\title{
COMUNICAÇÃO ELETRÔNICA ENTRE PROFISSIONAIS DE SAÚDE NA ASSISTÊNCIA AO PACIENTE: REVISÃO INTEGRATIVA
}

\author{
Electronic communication between health professionals in patient assistance: integrative review
}

\section{Comunicación electrónica entre profisionales de salud em la asistencia al paciente: revisión integrativa}

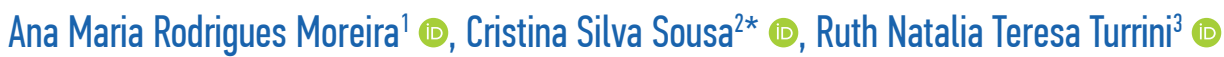

RESUMO: Objetivo: Analisar a literatura científica a respeito da comunicação por meios eletrônicos entre profissionais de saúde. Método: Revisão integrativa da literatura, realizada nas bases de dados e/ou portais PubMed, Biblioteca Virtual em Saúde e Cochrane, até agosto de 2018, com descritores combinados, que respondem à pergunta norteadora: "Como ocorre a comunicação eletrônica entre os profissionais de saúde na assistência ao paciente?囚. Resultados: Seis artigos foram incluídos, publicados de 2011 a 2016, no idioma Inglês. Os recursos foram smartphone, pager e tablet. Os aplicativos utilizados foram WhatsApp, Medigram e Serviço de Mensagens Curtas (SMS). Agilidade, facilidade de uso e auxílio na tomada de decisão foram as vantagens encontradas no uso desse recurso na comunicação dos profissionais; dentre as desvantagens estão ausência de privacidade e de confidencialidade das informações, interrupções na assistência e inabilidade no uso do recurso tecnológico. Conclusão: $\mathrm{O}$ uso de aplicativos para troca de mensagens e comunicação interdisciplinar de fato proporciona agilidade na comunicação, mas a confidencialidade desses dados ainda é uma questão a ser tratada. Assim, cabe ao enfermeiro conduzir a comunicação com os demais profissionais, preservando a privacidade do paciente.

Palavras-chave: Aplicativos móveis. Troca de informações. Smartphone. Comunicação. Enfermagem.

ABSTRACT: Purpose: To analyze scientific literature regarding the communication via electronic means between health professionals. Method: Integrative revision of the literature carried through databases and/or portals PubMed, Virtual Health Library and Cochrane, until August 2018, with combined describers, who answer to the leading question: "How does the electronic communication between health professionals in patient assistance occur?". Results: Six articles were included, published from 2011 to 2016 in the English language. Features were smartphone, pager and tablet. The applications used were WhatsApp, Medigram and Short Message Service (SMS). Agility, ease of use and support in decision-making were the advantages found in the use of this resource in the communication of professionals; among the disadvantages are lack of privacy and confidentiality of information, interruptions in assistance and inability to use the technological resource. Conclusion: The use of applications for messaging and interdisciplinary communication does indeed provide agility in communication, but the confidentiality of such data is still an issue to be addressed. Thus, it is up to the nurse to conduct communication with the other professionals, preserving the patient's privacy. Keywords: Mobile applications. Information exchange. Smartphone. Communication. Nursing.

RESUMEN: Objetivo: Analizar la literatura científica acerca de la comunicación por medios electrónicos entre profesionales de salud. Método: Revisión integrativa de la literatura, realizada en las bases de datos y/o portales PubMed, Biblioteca Virtual em Salud y Cochrane, hasta agosto de 2018, con descriptores combinados, que responden a la pregunta orientadora: “¿Cómo ocurre la comunicación electrónica entre los profesionales de salud en la asistencia al paciente?”. Resultados: Seis artículos fueron incluidos, publicados de 2011 a 2016, en el idioma Inglés. Los recursos fueron teléfono inteligente,

'Enfermeira. Especialista em Gestão em Serviços de Saúde pela Universidade Nove de Julho (Uninove) - São Paulo (SP), Brasil.

2Pós-Doutora em Enfermagem. Docente temporário do Departamento de Enfermagem Médico-Cirúrgico da Escola de Enfermagem da Universidade de São Paulo (USP) - São Paulo (SP), Brasil.

${ }^{3}$ Professora Associada do Departamento de Enfermagem Médico-Cirúrgico da Escola de Enfermagem da USP - São Paulo (SP), Brasil.

*Autor correspondente: thicoteco@bol.com.br

Recebido: 24/10/2018 - Aprovado: 24/04/2019

DOl: $10.5327 / 21414-4425201900020008$ 
pager y tablet. Las aplicaciones utilizadas fueron Whatsapp, Medigram y Servicio de mensajes cortos (SMS). Agilidad, facilidad de uso y ayuda em la tomada de decisión fueron las ventajas encontradas en el uso de ese recurso en la comunicación de los profesionales; entre las desventajas están la ausencia de privacidad y de confidencialidad de las informaciones, interrupciones em la asistencia e inhabilidad en el uso del recurso tecnológico. Conclusión: El uso de aplicaciones para el intercambio de mensajes y la comunicación interdisciplinaria de hecho proporciona agilidad en la comunicación, per la confidencialidad de estos datos sigue siendo una cuestión a tratar. Así, corresponde al enfermero conducir la comunicación con os demás profesionales, preservando la privacidad del paciente.

Palabras clave: Aplicaciones móviles. Intercambio de información. Teléfono inteligente. Comunicación. Enfermería.

\section{INTRODUÇÃo}

Com a expansão do uso de smartphones, combinada com o aumento da conectividade celular e do Wi-Fi, houve aumento significativo no uso de smartphones no ambiente hospitalar ${ }^{1}$.

No Brasil, publicou-se recentemente uma lei de proteção de dados pessoais, que prevê normas específicas sobre dados sensíveis. Ressalta-se a importância do chamado "princípio da precaução”, que prevê a inversão do ônus da prova, ou seja, quem pratica a ação que pode causar danos individuais ou coletivos deve apresentar as provas. Nos Estados Unidos da América, por exemplo, existe a Health Insurance Portability and Accountability Act (HIPAA), uma instituição com o intuito de proteger toda informação pessoal utilizada na prestação de serviços de saúde ${ }^{2}$.

No Brasil, o aplicativo mais comum na troca de mensagens é conhecido como WhatsApp (Whatsapp Inc. Mountain View, CA), um aplicativo de mensagens instantâneas para smartphones, que utiliza a internet para enviar mensagens de texto, imagens, vídeos, localização do usuário e mensagens de áudio ${ }^{3}$.

A comunicação impressa, como importante veículo de comunicação em massa, vem gradualmente perdendo espaço para as novas mídias, inclusive nos ambientes hospitalares, com os prontuários eletrônicos e a telemedicina. Assim, as possibilidades de comunicação se expandiram entre os profissionais de saúde, viabilizadas pela internet e pelo uso do smartphone.

Vivenciamos um ambiente profissional com a constante troca de informações por meio de mensagens. Por esses aplicativos, é possível compartilhar imagens e ter acesso imediato às respostas, com a facilidade de interação entre profissionais e pacientes.

Segundo a legislação do Conselho Federal de Medicina (CFM) $\mathrm{n}^{\circ} 1.643 / 2002$, as informações sobre o paciente identificado só podem ser transmitidas a outro profissional com prévia permissão, mediante seu consentimento livre e esclarecido e sob rígidas normas de segurança, capazes de garantir a confidencialidade e a integridade das informações ${ }^{4}$, no entanto a prática empírica mostra que a comunicação ocorre de modo indistinto, o que pode comprometer o sigilo de dados dos pacientes.

Nesse processo de comunicação por meios eletrônicos, interferências como ruídos e conversas paralelas podem ocasionar falhas na transmissão da informação; e essas falhas podem trazer prejuízos diretos para a assistência prestada ao paciente 5 .

É importante assumir um olhar crítico sobre a utilização do smartphone na comunicação entre os profissionais, para melhor conhecer o impacto positivo e negativo ao paciente, pois não raro a mídia relata casos de violação de privacidade e de sigilo na área da saúde.

Dada a carência de estudos de revisão sobre esse meio de comunicação e a expansão da utilização da mídia eletrônica na área da saúde, justifica-se a presente revisão integrativa da literatura.

\section{OBJETIVO}

Analisar a literatura científica a respeito da comunicação por meios eletrônicos entre profissionais de saúde.

\section{MÉTODO}

Trata-se de uma revisão integrativa da literatura, um método de pesquisa utilizado desde a década de 1980, norteada pela prática baseada em evidências. Tem por objetivo a integração entre a pesquisa científica e a prática profissional, possibilita a síntese do estado do conhecimento de um assunto e pode apontar lacunas do conhecimento que merecem ser investigadas ${ }^{6}$.

Esse tipo de revisão propõe as seguintes etapas: formulação de uma questão norteadora, busca na literatura dos estudos relacionados ao tema, categorização, avaliação, inclusão, 
interpretação, resultados e síntese do conhecimento evidenciado nos artigos analisados ${ }^{6}$. Assim, este estudo apresenta como pergunta norteadora: "Como ocorre a comunicação eletrônica entre os profissionais de saúde na assistência ao paciente?".

Estabeleceram-se como critérios de inclusão: estudos que abordam a comunicação entre profissionais de saúde, publicados nos idiomas inglês, espanhol, português, francês e italiano. Os critérios de exclusão foram: estudos que abordam comunicação entre pacientes e profissionais de saúde, gestão hospitalar, monitoramento de sinais e sintomas.

A busca foi realizada em bases de dados e/ ou portais: National Library of Medicine (PubMed, PMC), Biblioteca Virtual em Saúde (BVS) e Cochrane ${ }^{7}$. Como a base de dados mais antiga, Medical Literature Analysis and Retrieval System Online (Sistema Online de Busca e Análise de Literatura Médica - MEDLINE), incorpora artigos desde 1966, considerou-se como período de busca desse ano a agosto de 2018.

Utilizaram-se descritores não controlados e operadores booleanos na estrutura de busca: (smartphone) AND (communication) AND (health team OR health staff) NOT (pain OR diabetic OR health promotion OR mental health). Nas bases de dados sem interface com essa estratégia, utilizou-se a mesma estrutura com o termo booleano AND para fazer a ligação entre as caixas de busca. Destaca-se que foram realizadas inúmeras combinações com descritores controlados, que resultou em elevado quantitativo de estudos, inviabilizando a análise. Assim, para permitir uma análise de acordo com os critérios estabelecidos, o uso de descritores não controlados foi fundamental para a seleção dos estudos.

Foram localizados 655 artigos nas bases de dados. Inicialmente, realizou-se uma análise dos títulos de acordo com o critério de inclusão, excluindo-se 613 artigos por não atenderem à pergunta norteadora. Posteriormente, os 42 artigos remanescentes foram avaliados pelos resumos e restaram 6 artigos para a leitura na íntegra (Figura 1).

Para a coleta de dados, foi elaborado um instrumento com informações acerca de: autores, título do artigo, ano, local, objetivo, tipo de estudo, população, características da amostra, tipo de equipamento e aplicativo, critérios de inclusão e conclusões. Os dados foram analisados com base em uma tabela-síntese. Para avaliação metodológica dos estudos selecionados, utilizou-se o nível de evidência Oxford ${ }^{7}$. Para apresentação da síntese dos artigos foi construído um quadro sinóptico contendo as seguintes características: autores / ano, tipo de estudo, tipo de instituição/país, tipo de equipamento/comunicação, população/número de participantes, tempo de observação, objetivo do estudo e nível de evidência.

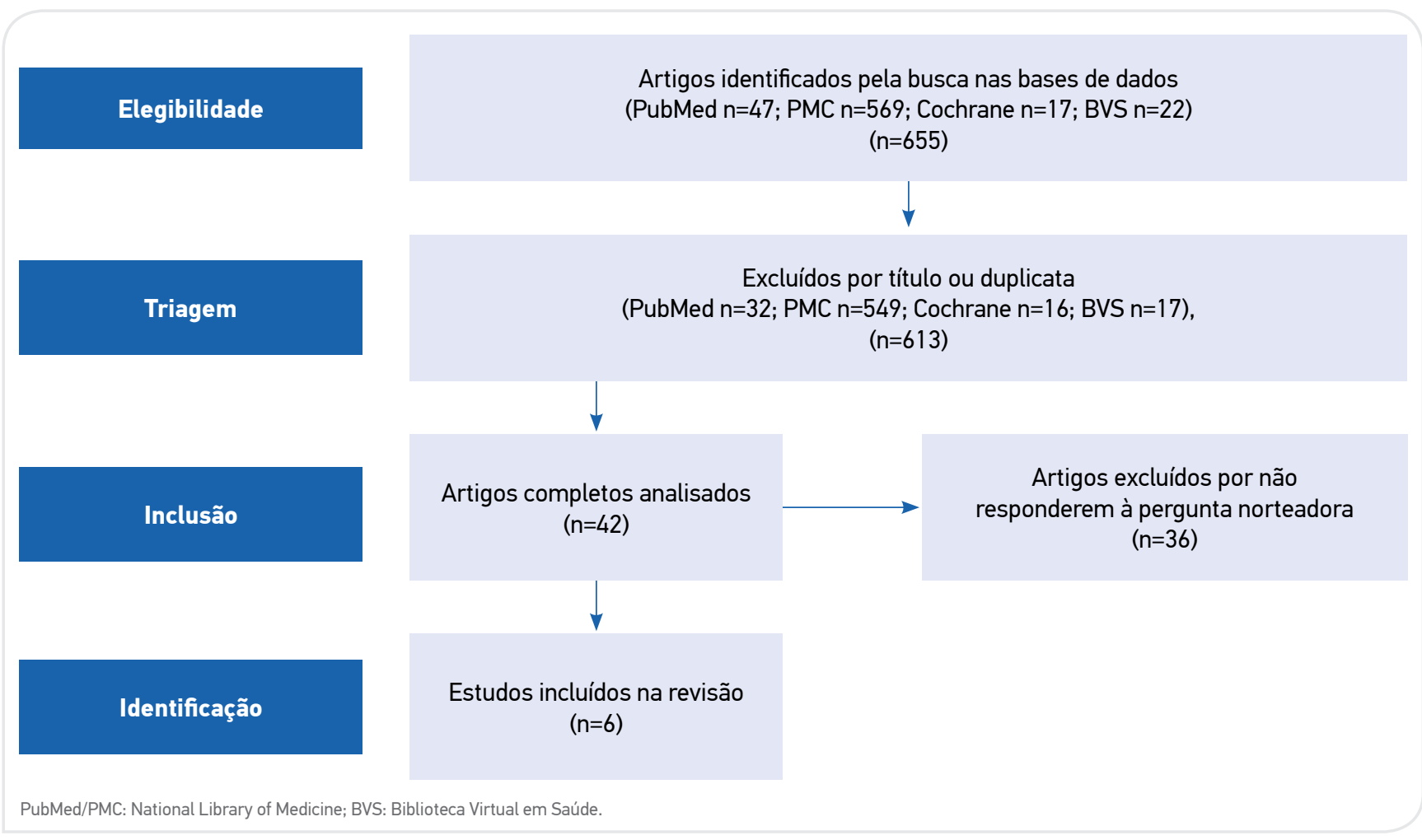

Figura 1. Diagrama da busca nas bases de dados e/ou portais. 


\section{RESULTADOS}

Os seis artigos selecionados foram publicados no período de 2011 a 2016. Dentre eles, dois artigos resultaram de pesquisa realizada nos Estados Unidos ${ }^{8,9}$, três no Canadá ${ }^{10-12} \mathrm{e}$ um estudo de base populacional no Quênia ${ }^{13}$ (Quadro 1).

Dois estudos foram quantitativos transversais do tipo survey $^{9,10}$, um ensaio clínico randomizado ${ }^{8}$, um estudo qualitativo com método etnográfico ${ }^{10} \mathrm{e}$ dois com análise quanti-qualitativa por análise de conteúdo ${ }^{11,13}$. O tempo de observação, quando descrito, variou entre 8 semanas e 17 meses.

A população de estudo nos artigos incluiu médicos assistentes ${ }^{8,10,11}$, coordenadores de grupos clínicos, médicos residentes $^{8-12}$, médicos internistas ${ }^{9}$, estudantes de medicina ${ }^{10}$, enfermeiros ${ }^{10,11}$, farmacêuticos ${ }^{8}$, agentes comunitários de saúde ${ }^{13}$, supervisores de grupos de agentes comunitários de saúde $^{13}$, profissionais do Ministério da Saúde local ${ }^{13}$, profissionais ligados a organizações não governamentais ou instituições acadêmicas participantes do projeto ${ }^{13}$.

O equipamento utilizado para comunicação eletrônica em cinco estudos foi o smartphone $e^{9-11}$, e, em outro, o pager e o tablet $^{8}$. Os estudos analisaram formas diferentes de comunicação: aplicativos de troca de mensagens, como WhatsApp $p^{12,13}$ e Medigram ${ }^{8}$, próprio para profissionais de saúde; e-mail pelo servidor do Blackberry ${ }^{10,11}$, serviço de mensagem eletrônica pelo $\mathrm{SMS}^{9}$ e mensagens pelo pager ${ }^{8}$.

O uso do smartphone ocorreu de diferentes maneiras: comunicação dos profissionais em ambiente intra-hospitalar ${ }^{8}$, comunicação e educação do paciente ${ }^{9,10}$, trocas de imagens e vídeos do paciente entre residentes e médicos assistentes para auxílio diagnóstico ${ }^{9,10}$, comunicação eletrônica entre enfermeiros e médicos para transmitir informação e solicitar ação do médico ${ }^{10,11}$, mensagens e fotos entre agentes comunitários e supervisores de saúde para orientação na conduta ${ }^{13}$.

As trocas de mensagens ocorreram entre residentes e preceptores médicos durante a avaliação do paciente, para relatar alterações e esclarecer dúvidas ${ }^{8,9,12}$; e entre médicos e enfermeiros, para relatar alterações clínicas ou obter informações acerca do paciente ${ }^{10,11}$. Alguns autores mencionaram o quantitativo de mensagens analisadas: $12.936^{10}, 13.717$ chamadas $^{10,11}$ e 1.830 posts $^{13}$.

A maior ocorrência de trocas de mensagens ocorreu durante as visitas médicas e na liberação de resultados de exames com alterações laboratoriais ou de imagem, principalmente na necessidade de outros profissionais para avaliação a distância ${ }^{8-11}$.
Os estudos foram realizados em instituições de saúde/ ensino: Universidade de Toronto ${ }^{12}$ e quatro hospitais de ensino: Hospital Geral em Toronto (Western), Brigham and Women's Hospital Massachusetts, University of Utah e Stanford Hospital ${ }^{8,9,11}$. Um estudo envolveu agentes comunitários de atenção primária à saúde e servidores, participantes de um projeto de ensino a distância, por meio de fórum em Makueni e Kibera, no Quênia ${ }^{13}$.

Foram identificadas vantagens na utilização da comunicação eletrônica: agilidade na troca de informações, uma vez que a maioria dos participantes tinha por hábito utilizar o recurso do smartphone também para comunicação pessoal ${ }^{12,13}$; benefício na educação a distância com o aplicativo WhatsApp como ferramenta de melhoria em ambientes com expressivos problemas sociodemográficos e na tutoria de profissionais para a resolução de problemas, no caso, um surto de cólera ${ }^{13}$; possibilidade de fóruns de educação em saúde a distância ${ }^{13}$; disponibilidade de aplicativos de cálculos de medicações e outros que auxiliam na tomada de decisão pelos profissionais de saúde 9 .

Com relação à percepção sobre a utilização de aplicativos para comunicação interprofissional, foram relatados aspectos positivos, como agilidade na troca de informação ${ }^{12,13}$; conhecimento por parte dos médicos dos nomes das enfermeiras responsáveis pelos seus pacientes ${ }^{10}$; maior eficiência dos residentes no trabalho clínico e percepção de melhor cuidado $^{8,9,11,12}$.

Apesar dos estudos terem avaliado a utilização desses equipamentos como uma poderosa ferramenta de comunicação ${ }^{8-11}$, alguns aspectos foram apontados como motivo de preocupação. Entre eles, a privacidade e a confidencialidade das informações relacionadas ao paciente; interrupções frequentes durante o atendimento médico (cerca de $46 \%)^{9,12} \mathrm{e}$ durante atividades de ensino ${ }^{10,12}$; incompatibilidade nas respostas entre médicos; frustração relatada por enfermeiros, pois não recebiam retorno em cerca de $50 \%$ de mensagens enviadas, o que requeria novos chamados telefônicos ${ }^{10,11}$; adesão à utilização para fins específicos com o paciente, incluindo calculadoras, sistemas de pontuação, diagnóstico e registro médico?.

Fragilidades na comunicação também foram relatadas, como pouco retorno na chamada do enfermeiro por divergência nos critérios de criticidade da situação ${ }^{10}$; residentes relataram que $42 \%$ dos e-mails das enfermeiras não necessitavam de respostas, pois eram apenas informativos ${ }^{11}$; e falta de habilidade no uso do smartphone e aplicativos ${ }^{8,9}$. 
Quadro 1. Síntese dos seis estudos selecionados como amostra da presente pesquisa.

\begin{tabular}{|c|c|c|c|c|c|c|c|}
\hline $\begin{array}{c}\text { Autores/ } \\
\text { ano }\end{array}$ & $\begin{array}{c}\text { Tipo } \\
\text { de estudo }\end{array}$ & $\begin{array}{c}\text { Tipo de } \\
\text { instituição, } \\
\text { país }\end{array}$ & \begin{tabular}{c|} 
Tipo de \\
equipamento, \\
comunicação
\end{tabular} & $\begin{array}{l}\text { População } \\
\text { ( } n^{\circ} \text { de } \\
\text { participantes) }\end{array}$ & $\begin{array}{c}\text { Tempo de } \\
\text { observação }\end{array}$ & $\begin{array}{l}\text { Objetivo } \\
\text { do estudo }\end{array}$ & Evidência \\
\hline $\begin{array}{l}\text { Przybylo } \\
\text { et al. } \\
(2014)^{8}\end{array}$ & $\begin{array}{l}\text { Ensaio clínico } \\
\text { randomizado }\end{array}$ & $\begin{array}{l}\text { Stanford } \\
\text { Hospital, } \\
\text { Estados } \\
\text { Unidos }\end{array}$ & $\begin{array}{l}\text { Smartphone, } \\
\text { Medigram } \\
\text { compliant } \\
\text { group } \\
\text { messaging } \\
\text { (HCGM) e } \\
\text { sistema pager } \\
\text { tradicional }\end{array}$ & $\begin{array}{l}\text { Participantes } \\
\text { divididos em } \\
\text { cinco grupos de } \\
\text { medicina interna: } \\
\text { três randomizados } \\
\text { para o grupo de } \\
\text { estudo e dois para } \\
\text { o grupo controle } \\
(n=75)\end{array}$ & 8 semanas & $\begin{array}{l}\text { Determinar se a inclusão } \\
\text { de um aplicativo de } \\
\text { mensagens de texto } \\
\text { Medigram ao pager } \\
\text { unidirecional melhora } \\
\text { a comunicação entre a } \\
\text { equipe hospitalar }\end{array}$ & $1 \mathrm{~B}$ \\
\hline $\begin{array}{l}\text { Raaum } \\
\text { et al. } \\
(2015)^{9}\end{array}$ & $\begin{array}{l}\text { Estudo } \\
\text { transversal } \\
\text { tipo survey }\end{array}$ & $\begin{array}{l}\text { University of } \\
\text { Utah, } \\
\text { Brigham and } \\
\text { Women's } \\
\text { Hospital, } \\
\text { Estados } \\
\text { Unidos }\end{array}$ & $\begin{array}{l}\text { Smartphone, } \\
\text { SMS }\end{array}$ & $\begin{array}{l}\text { Médicos } \\
\text { residentes } \\
(175 / 330) \\
(n=175)\end{array}$ & $\begin{array}{c}\text { Não } \\
\text { informado }\end{array}$ & $\begin{array}{l}\text { Caracterizar o uso } \\
\text { de smartphone por } \\
\text { médicos residentes } \\
\text { em instituições } \\
\text { acadêmicas e investigar } \\
\text { seu treinamento em } \\
\text { uso clínico desses } \\
\text { dispositivos }\end{array}$ & $2 \mathrm{C}$ \\
\hline $\begin{array}{l}\text { Wu et al. } \\
(2011)^{10}\end{array}$ & $\begin{array}{l}\text { Estudo } \\
\text { qualitativo com } \\
\text { referencial } \\
\text { etnográfico }\end{array}$ & $\begin{array}{l}\text { Hospital geral } \\
\text { de ensino } \\
\text { em Toronto, } \\
\text { Canadá }\end{array}$ & $\begin{array}{c}\text { Telefone celular } \\
\text { tipo Blackberry, } \\
\text { e-mail }\end{array}$ & $\begin{array}{l}\text { Médicos } \\
\text { residentes em } \\
\text { clínica médica e } \\
\text { enfermeiras } \\
(n=34)\end{array}$ & 17 meses & $\begin{array}{l}\text { Avaliar o padrão de } \\
\text { uso do smartphone, } \\
\text { as vantagens e } \\
\text { desvantagens de seu } \\
\text { uso; determinar aspectos } \\
\text { para melhorar o seu uso }\end{array}$ & $2 C$ \\
\hline $\begin{array}{l}\text { Smith } \\
\text { et al. } \\
(2012)^{11}\end{array}$ & $\begin{array}{l}\text { Estudo } \\
\text { transversal } \\
\text { quanti- } \\
\text { qualitativo de } \\
\text { e-mail }\end{array}$ & $\begin{array}{l}\text { Enfermarias } \\
\text { clínicas de } \\
\text { hospital geral } \\
\text { em } \\
\text { Toronto, } \\
\text { Canadá }\end{array}$ & $\begin{array}{c}\text { Telefone celular } \\
\text { tipo Blackberry, } \\
\text { e-mail }\end{array}$ & $\begin{array}{l}\text { Médicos } \\
\text { residentes de } \\
\text { clinica médica } \\
(34 / 67) \\
(n=34)\end{array}$ & 6 meses & $\begin{array}{l}\text { Analisar o conteúdo } \\
\text { da comunicação por } \\
\text { e-mail entre médicos, } \\
\text { enfermeiros e outros } \\
\text { profissionais de saúde }\end{array}$ & $2 C$ \\
\hline $\begin{array}{l}\text { Tran } \\
\text { et al. } \\
(2014)^{12}\end{array}$ & $\begin{array}{c}\text { Estudo } \\
\text { transversal tipo } \\
\text { survey }\end{array}$ & $\begin{array}{l}\text { Faculdade de } \\
\text { Medicina da } \\
\text { Universidade } \\
\text { de Ontário, } \\
\text { Canadá }\end{array}$ & $\begin{array}{l}\text { Smartphones, } \\
\text { WhatsApp }\end{array}$ & $\begin{array}{l}\text { Estudantes de } \\
\text { medicina do } 4^{\circ} \\
\text { ano }(99 / 218) \\
(n=99)\end{array}$ & $\begin{array}{c}\text { Não } \\
\text { informado }\end{array}$ & $\begin{array}{l}\text { Avaliar o uso de } \\
\text { smartphones durante } \\
\text { o estágio clínico e } \\
\text { descrever a percepção de } \\
\text { impacto na privacidade } \\
\text { e confidencialidade das } \\
\text { informações de saúde }\end{array}$ & $2 C$ \\
\hline $\begin{array}{l}\text { Henry } \\
\text { et al. } \\
(2016)^{13}\end{array}$ & $\begin{array}{c}\text { Estudo } \\
\text { transversal } \\
\text { quantiqualitativo } \\
\text { de } e \text {-mail }\end{array}$ & $\begin{array}{l}\text { Duas } \\
\text { unidades } \\
\text { do estudo } \\
\text { mobile } \\
\text { learning } \\
\text { intervention } \\
\text { (mCHW), no } \\
\text { Quênia }\end{array}$ & $\begin{array}{l}\text { WhatsApp, } \\
\text { fórum } \\
\text { aprendizado }\end{array}$ & $\begin{array}{l}\text { Agentes } \\
\text { comunitários } \\
\text { de saúde, } \\
\text { supervisores } \\
\text { do projeto, } \\
\text { funcionários } \\
\text { de entidades } \\
\text { de saúde do } \\
\text { governo, de } \\
\text { organizações não } \\
\text { governamentais } \\
\text { ou instituições } \\
\text { parceiras } \\
\text { (n=41) }\end{array}$ & 6 meses & $\begin{array}{l}\text { Documentar o uso do } \\
\text { WhatsApp como suporte } \\
\text { para supervisão dos } \\
\text { agentes comunitários; } \\
\text { identificar como isso } \\
\text { é feito e como isso } \\
\text { se relaciona com a } \\
\text { supervisão geral do } \\
\text { projeto }\end{array}$ & $2 C$ \\
\hline
\end{tabular}

SMS: Sistema de Mensagens Curtas. 
O estudo que teve por objetivo comparar dois sistemas de mensagens, um de acordo com a lei de proteção à informação, o Medigram (aplicativo de acesso livre para smartphone com senha de acesso) e um pager de uso no hospital de estudo, não observou diferença estatística em relação aos aspectos das mensagens recebidas em cada modalidade; mas quanto à efetividade percebida nos dois modos de comunicação, $\mathrm{o}$ Medigram foi mais bem avaliado ${ }^{8}$. Os recursos mais efetivos do Medigram foram: facilidade de uso, capacidade de comunicação por mensagens no grupo, velocidade. Os recursos menos efetivos mencionados foram: capacidade para estar em todos os lugares ao mesmo tempo (ubiquidade), uso inconsistente por aqueles que acessavam o aplicativo e confiabilidade na transmissão da mensagem ${ }^{8}$.

Os autores de um dos estudos ${ }^{12}$ abordaram a questão ética dessa forma de comunicação e observaram que $78 \%$ dos residentes referiram nunca identificar o paciente durante a troca de mensagens sobre conduta clínica e 59\% desses profissionais afirmaram ter recebido, em sua formação, orientações sobre como utilizar o recurso de comunicação eletrônica e preservar a confidencialidade sobre dados do paciente ${ }^{13}$.

De modo geral, os resultados demonstram que a comunicação eletrônica traz benefícios, mas deve haver prudência.

\section{DISCUSSÃO}

Poucos estudos responderam à questão norteadora, o que pode ser decorrente do pouco tempo de uso desse recurso no ambiente profissional, pois a publicação mais antiga foi de 2011. Os estudos inseridos têm nível de evidência predominante $2 \mathrm{C}$. Também não foram encontrados estudos nacionais com a temática.

O uso do BlackBerry foi superado pelo iPhone ${ }^{\circledR} \mathrm{em} 2010$, e extinto em 2016. Com isso, estudos atuais retratam outros sistemas de smartphone como IOS, Android ou Windows Phone. Entretanto, indiferentemente do recurso tecnológico utilizado, os benefícios evidenciados no uso da ferramenta de comunicação são semelhantes aos achados deste estudo: agilidade na comunicação e eficiência no trabalho clínico, devido ao compartilhamento do caso e à troca de informações sobre conduta clínica ${ }^{14,15}$.

O sistema de mensagens do smartphone (SMS) é semelhante ao WhatsApp, na troca de mensagens de texto, mas passou a de ser menos utilizado com a chegada desse novo aplicativo.

Dois estudos ${ }^{12,13}$ fizeram uso do aplicativo WhatsApp para troca de mensagens entre os profissionais de saúde. Esse recurso tem se mostrado uma ferramenta barata e ágil, que pode ser utilizada em qualquer sistema de telefonia móvel, com fácil manuseio, rapidez e agilidade no retorno de respostas. Por outro lado, há falta de compreensão de como a informação é transmitida e armazenada ao usar o WhatsApp e problemas com a confidencialidade do paciente e a segurança dos dados ${ }^{14}$.

Os benefícios adicionais do WhatsApp incluem a capacidade de criar mensagens de grupo, permitindo melhor comunicação da equipe, revisão de conversas na saída e opiniões adicionais sobre casos e gerenciamento de pacientes ${ }^{1,16}$.

Observa-se, na prática clínica, o uso de grupos em WhatsApp para enfermeiros e coordenadores de área para rápida comunicação de informações relacionadas à rotina de trabalho, escalas e orientações. Outro perfil de grupos é composto de enfermeiros de vários hospitais para troca de informações de rotinas e orientações de materiais.

Esse tipo de comunicação facilita a transmissão de mensagens e pode ser visualizado rapidamente; entretanto, aumenta o uso do smartphone no ambiente de trabalho.

O uso do aplicativo Medigram foi identificado somente em um estudo ${ }^{8}$. As mensagens são criptografadas e o acesso ao aplicativo é protegido por senha. Esse recurso tem sido utilizado no espaço de trabalho da área de saúde, demonstrando aumentar a responsabilidade, melhorar a eficiência, a integração do fluxo de trabalho e a satisfação geral ${ }^{1}$. Há outro aplicativo disponível no mercado, o Vocera, para comunicação intra-hospitalar, seguro e com privacidade dos dados compartilhados ${ }^{17}$.

Os recursos de troca de mensagens exigem cuidado durante a digitação, pois são comuns erros, abreviações, além de corretores automáticos, que distorcem informações. Subsequentes correções geram inúmeras mensagens, que possibilitam a perda de informações importantes, por passarem despercebidas na leitura. Há também o erro no envio e no recebimento de mensagens; ausência de rede de internet; esquecimento, perda ou roubo do smartphone, que impedem o contato com o profissional. Assim, essa não deve ser a única ferramenta de comunicação ${ }^{18}$.

As desvantagens desses recursos de comunicação também foram observadas em outros estudos, como: interrupção das atividades laborais para consultar e responder mensagens, atender ligações ${ }^{16,17} \mathrm{e}$ a possível exposição do paciente ${ }^{16}$.

O uso de smartphone no ambiente de trabalho pode ser reconhecido como uma ferramenta indispensável para completar as tarefas diárias e certas rotinas clínicas ${ }^{19}$, no entanto há preocupação no desempenho no trabalho, pois cerca de 
$70 \%$ dos enfermeiros testemunharam o uso inadequado do smartphone durante as atividades laborais ${ }^{20}$.

A presente revisão também identificou que enfermeiros se mostram preocupados com o uso dos smartphones ${ }^{8,10,12}$. Houve relatos de incidentes ocasionados pelo uso do aparelho durante a assistência e reclamações de pacientes quanto ao profissional com uso excessivo, em um estudo realizado com estudantes e enfermeiros ${ }^{21}$.

Profissionais da saúde, como médicos e enfermeiros, mostraram-se extremamente incomodados com as interrupções no fluxo de trabalho durante a assistência por ligações e mensagens eletrônicas ${ }^{22}$.

Em um estudo ${ }^{23}$, alunos de graduação em enfermagem mencionaram que observaram $83 \%$ dos enfermeiros utilizando o smartphone durante a prática clínica e $15 \%$ deles disseram que deveria haver políticas de regulação para o uso do aparelho. Por outro lado, os alunos, durante a graduação, são encorajados a usar o smartphone para completar os conteúdos de aprendizado ${ }^{23}$. Enfermeiros assistenciais, ao serem questionados a respeito do uso do smartphone, relataram que esses equipamentos trazem benefícios à assistência prestada ao paciente, aplicando a tecnologia em favor das boas práticas ${ }^{21}$.

Entre as limitações deste estudo, estão os poucos artigos publicados sobre o uso desse recurso na comunicação entre profissionais de saúde, o que limita a avaliação de seu uso, além da variedade de delineamentos de pesquisa. Os resultados permitiram identificar a necessidade de estudos que discutam as questões éticas envolvidas nesse processo de troca de mensagens, segurança e confidencialidade de dados.

\section{CONCLUSÃO}

Conclui-se que há pouca literatura, até o momento, sobre a temática de comunicação eletrônica entre profissionais de saúde. Fica evidente a vantagem da agilidade da informação e a possibilidade de criação de grupos de conversa com maior disseminação de orientações e rotinas entre os profissionais. Contudo, ainda existe um longo caminho a ser percorrido, devido a demandas legais e éticas, quando essas informações estão relacionadas aos pacientes. Os profissionais de saúde ainda não têm critérios definidos sobre a troca de informações sigilosas dos pacientes e devem estar atentos às interrupções e aos possíveis incidentes relacionados ao uso do smartphone no ambiente de trabalho.

É desejado que o enfermeiro seja o protagonista em conduzir e transmitir as informações do paciente, identificar as informações pertinentes a serem transmitidas à equipe multidisciplinar, manter a privacidade das informações de forma ética e respeitosa e gerenciar a assistência para que o paciente seja beneficiado pelo uso da comunicação eletrônica, em favor de sua recuperação.

\section{REFERÊNCIAS}

1. Pourmand A, Roberson J, Gallugi A, Sabha Y, O'Connell. Secure smartphone application-based text messaging in emergency department, a system implementation and review of literature. Am J Emerg Med. 2018;36(9):1680-5. https://doi.org/10.1016/j.ajem.2018.06.067

2. Brasil. Lei no 13.709 , de 14 de agosto de 2018. Dispõe sobre a proteção de dados pessoais e altera a Lei $n^{\circ} 12.965$, de 23 de abril de 2014 (Marco Civil da Internet). Diário Oficial da União [Internet]. 2018 [acessado em 30 set. 2018];Seção l(157):59-64. Disponivel em: http://www.planalto. gov.br/ccivil_03/_Ato2015-2018/2018/Lei/L13709.htm

3. Petruzzi M, Benedittis M. WhatsApp: a telemedicine platform for facilitating remote oral medicine consultation and improving clinical examinations. Oral Surg Oral Med Oral Pathol Oral Radiol. 2016;121(3):248-54. https://doi.org/10.1016/j.000o.2015.11.005

4. Conselho Federal de Medicina. Resolução CFM no 1.643/2002. Define a disciplina a proteção de serviços através da telemedicina. Diário
Oficial da União [Internet]. 2002 [acessado em 30 set. 2018];Seção |:205. Disponível em: http://www.portalmedico.org.br/resolucoes/ cfm/2002/1643_2002.pdf

5. Sousa CS, Souza RCS, Gonçalves MC, Diniz TRZ, Cunha ALM. Comunicação efetiva entre o centro cirúrgico e a unidade de terapia intensiva. Rev SOBECC. 2014;19(1):44-50. https://doi.org/10.4322/ sobecc. 2014.004

6. Mendes KDS, Silveira RCCP, Galvão CM. Revisão integrativa: método de pesquisa para a incorporação de evidências na saúde e na enfermagem. Texto Contexto Enferm. 2008;17(4):758-64. http:// dx.doi.org/10.1590/S0104-07072008000400018

7. Centre for Evidence-Based Medicine. Levels of evidence [Internet]. Oxford: Centre for Evidence-Based Medicine; 2009 [acessado em 30 set. 2018]. Disponível em: http://www.cebm.net/oxford-centreevidence-based-medicine-levels-evidence-march-2009/ 
8. Przybylo JA, Wang A, Loftus P, Evans KH, Chu I, Shieh L. Smarter hospital communication: secure smartphone text messaging improves provider satisfaction and perception of efficacy, workflow. J Hosp Med. 2014;9(9):573-8. https://doi.org/10.1002/jhm.2228

9. Raaum SE, Arbelaez C, Vallejo CE, Patino Am, Colbert-Getz JM, Milne CK. Emergency medicine and internal medicine trainees' smartphone use in clinical settings in the United States. J Educ Eval Health Prof. 2015;12:48. http://dx.doi.org/10.3352/jeehp.2015.12.48

10. Wu R, Rossos P, Quan S, Reeves S, Lo V, Wong B, et al. An evaluation of the use of smartphone to communicate between clinicians: a mixed-methods study. J Med Internet Res. 2011;13(3):e.59. https:// doi.org/10.2196/jmir.1655

11. Smith CN, Quan SD, Morra D, Rossos PG, Khatibi H, Lo V, et al. Understanding interprofessional communication: a content analysis of email communications between doctors and nurses. Appl Clin Inf. 2012;3(1):38-51. http://dx.doi.org/10.4338/ACl-2011-11-RA-0067

12. Tran K, Morra D, Lo V, Quan SD, Abrams H, Wu RC. Medical students and personal smartphones in the clinical environment: the impact on confidentiality of personal health information and professionalism. J Med Internet Res. 2014;16(5):e132. https://doi.org/10.2196/jmir.3138

13. Henry JV, Winters N, Lakati A, Oliver M, Geniets A, Mbae SM, et al. Enhancing the supervision of community health workers with WhatsApp mobile messaging: qualitative findings from 2 low-resource settings in Kenya. Glob Health Sci Pract. 2016;4(2):311-25. https:// doi.org/10.9745/GHSP-D-15-00386

14. Popovici I, Morita PP, Doran D, Lapinsky S, Morra D, Shier A, et al.Technological aspects of hospital communication challenges: an observational study. Int J Qual Health Care. 2015;27(3):183-8. https:// doi.org/10.1093/intqhc/mzv016

15. Ganasegeran K, Renganathan P, Rashid A, Al-Dubai SA. The m-health revolution: exploring perceived benefits of WhatsApp use in clinical practice. Int J Med Inform. 2017;97:145-51. http://dx.doi.org/10.1016/j. ijmedinf.2016.10.013
16. Boulos MNK, Giustini DM, Wheeler S. Instagram and Whatsapp in health and healthcare: an overview. Future Internet. 2016;8(3):37. https://doi.org/10.3390/fi8030037

17. Webb C, Spina SP, Young S. Integrating smartphone communication strategy and technology into clinical practice: a mixed methods research study. Health Policy and Technology. 2016;5(4):370-5. http://dx.doi.org/10.1016/j.hlpt.2016.07.004

18. Redelmeier DA, Detsky AS. Pittalls with Smartphones in Medicine. J Gen Intern Med. 2013;28(10):1260-3. https://doi.org/10.1007/ s11606-013-2467-4

19. Putzer GJ, Park Y. The effects of innovation factors on smartphone adoption among nurses in community hospitals. Perspect Health Inf Manag [Internet]. 2010 [acessado em 30 ago. 2018];7:1b. Disponível em: https://www.ncbi.nlm.nih.gov/pmc/articles/PMC2805554/pdf/ phim0007-0001b.pdf

20. McBride D, LeVasseur SA, Li D. Nursing performance and mobile phone use: are nurses aware of their performance decrements? JMIR Hum Factors. 2015;2(1):e6. http://dx.doi.org/10.2196/humanfactors.4070

21. McNally G, Frey R, Crossan M. Nurse manager and student nurse perceptions of the use of personal smartphones or tablets and the adjunct applications, as an educationaltool in clinical settings. Nurse Educ Pract. 2017;23:1-7. http://dx.doi.org/10.1016/j.nepr.2016.12.004

22. Locke KA, Duffey-Rosenstein B, De Lio G, Morra D, Hariton N. Beyond paging: building a web-based communication tool for nurse and physicians. J Gen Interm Med. 2009;24(1):105-10. http://dx.doi. org/10.1007/s11606-008-0827-2

23. Cho $\mathrm{S}$, Lee E. Distraction by smartphone use during clinical practice and options about smartphone restriction policies: a cross-sectional descriptive study of nursing students. Nurse Educ Today. 2016;40:12833. http://dx.doi.org/10.1016/j.nedt.2016.02.021 